\title{
Dynamics of nearby groups of galaxies: the role of the cosmological constant
}

\author{
S. Peirani ${ }^{1}$ and J. A. de Freitas Pacheco ${ }^{2}$ \\ 1 Institut d'Astrophysique de Paris, 98bis Bd. Arago, 75014 Paris, France, Unité mixte de recherche 7095 CNRS, \\ Université Pierre et Marie Curie, \\ e-mail: peirani@iap.fr \\ 2 Observatoire de la Côte d'Azur, Laboratoire Cassiopée, UMR 6202, BP 4229, 06304 Nice Cedex 4, France \\ e-mail: pacheco@oca.eu
}

Received 4 March 2008 / Accepted 16 June 2008

\section{ABSTRACT}

\begin{abstract}
Context. Different cosmological data are consistent with the presence of an accelerated expansion of the Universe produced by an exotic matter-energy component, dubbed "dark-energy". A cosmological constant is a possible explanation for this expansion since it satisfies most of the observational constraints.

Aims. We investigate the consequences of such a component on the dynamics of galaxy groups by attempting to detect possible effects on scales of approximately few Mpc.

Methods. The Lemaitre-Tolman model was modified by the inclusion of the cosmological constant term and, from numerical solution of the equations of motion, a velocity-distance relation was obtained. This relation depends on two parameters: the central core mass and the Hubble parameter. The non-linear fit of this relation to available data enabled the masses of five nearby groups of galaxies and the Virgo cluster to be obtained as well as estimates of the Hubble constant.

Results. The analysis of the present results indicates that the velocity-distance relation derived from the modified Lemaitre-Tolman model in addition to that derived from the "canonical" model, provide equally acceptable fits to the existing available data. As a consequence, any robust conclusion about the effects of the cosmological constant on the dynamics of groups could be established. The mean value of the Hubble parameter derived from the present study of local flows is $H_{0}=65 \pm 7 \mathrm{~km} \mathrm{~s}^{-1} \mathrm{Mpc}^{-1}$.
\end{abstract}

Key words. galaxies: kinematics and dynamics - cosmology: cosmological parameters - methods: numerical

\section{Introduction}

Small groups of galaxies are common structures in the Universe and may contribute up to $\sim 50 \%$ of its matter content (Huchra \& Geller 1982; Geller \& Huchra 1983; Nolthenius \& White 1987). Early estimates of the mass-to-light $(M / L)$ ratios for groups, based on the virial relation, measured values of typically of approximately $170 M_{\odot} / L_{B, \odot}$ (Huchra \& Geller 1982). However, new and high quality data on galaxies situated in nearby groups (Karachentsev 2005 and references therein) yield values around 10-30 $M_{\odot} / L_{B, \odot}$, which are considerably smaller than previous estimates. If these new estimates are correct, then the local matter density would be only a fraction of the global matter density. The virial relation measures accurate masses only if groups are in dynamical equilibrium. In general, observers assume that this is the case if the crossing time, which is the ratio between a characteristic group radius and the typical velocity of galaxies belonging to the system, is less than the Hubble time. Using cosmological simulations, Niemi et al. (2007) identified groups with the same algorithm used by observers. Their analysis indicated that about $20 \%$ of them are not gravitationally bound and that this fraction increases when the apparent magnitude limit of the survey increases. Moreover, Niemi et al. (2007) did not find any correlation between the virial ratio $2 T /|W|$ and the crossing time, a result which is independent of the magnitude limit of the mock catalogue and weakens the criterium usually adopted to characterize the dynamical equilibrium.
Besides the question related to the mass estimates of groups, a second and long-standing problem concerns the fact that the dispersion in the peculiar velocities within the Hubble flow is quite small and is usually referred as the "coldness" of the local velocity flow (Sandage \& Tammann 1975; Giraud 1986; Schlegel et al. 1994; Ekholm et al. 2001). The presence of the dark energy was proposed as a possible explanation for the smoothness of the local Hubble flow (Chernin 2001; Teerikorpi et al. 2005). Dark matter simulations by Governato et al. (1997) for cosmological models with $\Omega_{\mathrm{m}}=1$ or $\Omega_{\mathrm{m}}=0.3$ are, according to these authors, unable to produce systems embedded in regions of "cold" flows, i.e., with $1 \mathrm{D}$ dispersion velocities of approximately $40-50 \mathrm{~km} \mathrm{~s}^{-1}$. From simulations based on a $\Lambda C D M$ cosmology, Macciò et al. (2005) and Peirani \& de Freitas Pacheco (2006, hereafter PP06) estimated values of the 1D dispersion velocity, averaged within a sphere of $\sim 3$ Mpc radius, of 80 and $73 \mathrm{~km} \mathrm{~s}^{-1}$ respectively. Hoffman et al. (2007) compared results from their own simulations of $C D M$ and $\triangle C D M$ cosmologies with identical parameters, apart from the presence or not of the cosmological constant term. They claimed that no significant differences were noticed in the velocity flow around galaxies having properties similar to those observed in the neighborhood of the Milky Way (MW).

In the case of groups consisting of a central and dominant mass or corresponding to a single or pair of massive galaxies and an external "cloud" of low mass satellites, an alternative approach to the virial relation was proposed by Lynden-Bell (1981) 
and Sandage (1986), based on the Lemaître-Tolman (LT) model (Lemaître 1933; Tolman 1934). The LT model provides a good description of the dynamics of a bound central core embedded in an extended halo, which approaches asymptotically a homogeneous Friedmann background. In this situation, three main distinct regions can be distinguished: i) the central core, in which the shell crossing has already occurred, generating energy exchanges that transform radial into random motions; ii) the zero-velocity surface, a boundary that separates infalling from expanding bound shells and iii) the "marginally" bound surface (zero total energy), segregating bound and unbound shells. Density profiles corresponding to the LT model were discussed by Olson \& Silk (1979) and an application of the model to the velocity field close to the Virgo cluster was made by for example Hoffman et al. (1980), Tully \& Shaya (1984) and Teerikorpi et al. (1992). The LT model, modified by the inclusion of a cosmological constant, was revisited by PP06, who applied their results to a sample of galaxies in the outskirts of the Virgo cluster and to dwarf galaxies in the vicinity of the M31-MW pair.

The velocity-distance $(v-R)$ relation is a photograph of the kinematic status of the different shells at a given instant. Such a relation, derived either from the "canonical" or the modified LT model, depends on only two parameters: the core mass and the Hubble parameter. Thus, if pairs of values $(v, R)$ are known for satellite galaxies of a given group, a non-linear fit can be performed between data and the theoretical $v-R$ relation, permitting a simultaneous determination of the core/central galaxy mass and of the Hubble parameter (PP06). In this paper, the $v-R$ relation, modified by the inclusion of a cosmological constant, was calculated for the present age of the universe by using an improved integration algorithm with respect to that employed originally by PP06. The fit of the resulting numerical values provides a slightly different solution, which was applied to data on the groups M81, Sculptor, CenA/M83, and IC342/Maffei-I, allowing their masses to be estimated as well as the Hubble parameter $H_{0}$. The previous analysis by PP06 of the Local Group and Virgo cluster was also revisited. As we shall see, the inclusion or not of the cosmological constant in the LT model does not affect considerably the resulting masses. However, the resulting values of the Hubble parameter are systematically higher for the LT model compared with those derived for the modified LT model, but mean values resulting from both approaches are consistent, within the uncertainties, with other independent determinations. This paper is organized as follows: in Sect. 2, a short review of the model is presented; in Sect. 3 an application to nearby groups is made, and, finally, in Sect. 4, our main conclusions are given.

\section{The model}

The spherical collapse model of a density perturbation originates in the work by Gunn \& Gott (1972), who described how a small spherical patch decouples from the homogeneous, expanding background, slows down, turns around, and collapses, forming finally a virialized system. The inclusion of other forms of energy besides gravitation has been the subject of many investigations as, for instance, those by Lahav et al. (1991), Wang \& Steinhardt (1998), and Maor \& Lahav (2005).

Here we follow the procedure adopted in our previous paper (PP06), since we intend to obtain, for the present age of the universe, the velocity profile of small galaxies affected by the gravitational field of the central massive object. This approach supposes that satellites do not contribute significantly to the total mass of the group, and that orbits are mainly radial and do not form a relaxed system. The effects of non-zero angular momentum orbits are discussed later. We also assume that displacements of the satellite galaxies, associated with the outer shells, develop at redshifts when the formation of the mass concentration around the core is almost complete (see, for instance, Peebles 1990) or, in other words, that any further mass accretion is neglected. Under these conditions, the equation of motion for a spherical shell of mass $m$, moving radially in the gravitational field created by a mass $M(\gg m)$, inside a shell of radius $R$, including the cosmological constant term is

$$
\frac{\mathrm{d}^{2} R}{\mathrm{~d} t^{2}}=-\frac{G M}{R^{2}}+\Omega_{\Lambda} H_{0}^{2} R
$$

This equation has a first integral, which expresses the energy conservation, given by

$\left(\frac{\mathrm{d} R}{\mathrm{~d} t}\right)^{2}=\frac{2 G M}{R}+\Omega_{\Lambda} H_{0}^{2} R^{2}+2 E$,

where $E$ is the energy per unit of mass of a given shell. We note that Eq. (2) is similar to the Hubble equation in which the energy plays the role of the curvature constant. To solve numerically Eq. (1), it is convenient to define the dimensionless variables $y=R / R_{0}, x=t H_{0}$, and $u=\dot{R} / H_{0} R_{0}$, where $R_{0}$ is the radius of the zero-velocity surface, i.e. $\dot{R}\left(R_{0}\right) \equiv v\left(R_{0}\right)=0$. In terms of these variables, the equations above can be rewritten as

$\frac{\mathrm{d}^{2} y}{\mathrm{~d} x^{2}}=-\frac{A}{2 y^{2}}+\Omega_{\Lambda} y$

and

$u^{2}=\frac{A}{y}+\Omega_{\Lambda} y^{2}+K$

where we have introduced the constants $A=2 G M /\left(H_{0}^{2} R_{0}^{3}\right)$ and $K=2 E /\left(H_{0} R_{0}\right)^{2}$. To integrate the equation of motion, initial conditions have to be imposed. We assume that the initial value of the shell radius equals $y_{i}=10^{-3}$, which corresponds to an initial proper dimension of about $1 \mathrm{kpc}$. When $y \ll 1$, the gravitational term dominates the right hand side of Eq. (3) and an approximate solution, valid for small values of the radius is $y \simeq(9 A / 4)^{1 / 3} x^{2 / 3}$, from which the initial value of the dimensionless time $x_{i}$, corresponding to the adopted value of $y_{i}$, can be estimated. Using the initial value $y_{i}$, the initial value of the velocity $u_{i}$ can be estimated from Eq. (4), for a given value of $K$, i.e. the shell energy. The constant $A$ is determined from the integration of Eq. (4) subject to the conditions $u(y=1)=0$ and

$x(y=1)=\int_{0}^{\infty} \frac{\mathrm{d} z}{(1+z) \sqrt{\Omega_{\Lambda}+\Omega_{m}(1+z)^{3}}}$,

which for the zero-velocity shell imply an energy $K=-\left(A+\Omega_{\Lambda}\right)$. We obtained $A=3.7683$ for $\Omega_{\Lambda}=0.7$, a value $3 \%$ higher than that obtained previously by PP06. Using the definition of $A$, the central mass can be estimated by the relation below, if the radius of the zero-velocity surface is known, namely,

$M=4.23 \times 10^{12} h^{2} R_{0}^{3} M_{\odot}$,

where $R_{0}$ is in Mpc and $h$ is the Hubble parameter in units of $100 \mathrm{~km} \mathrm{~s}^{-1} / \mathrm{Mpc}$. This result is essentially the basis of the method proposed by Lynden-Bell (1981) and Sandage (1986) to estimate the mass of the Local Group. The inclusion of the cosmological constant modifies the numerical factor and, as emphasized by PP06, masses derived by this procedure are, for the 


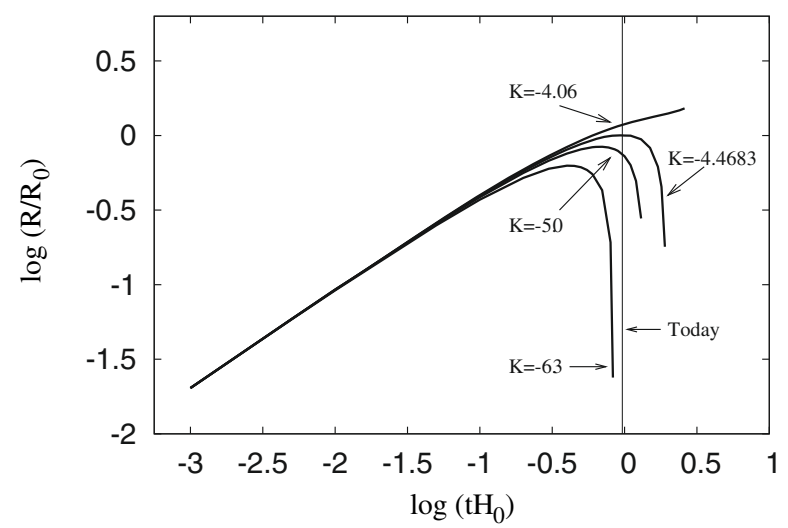

Fig. 1. Evolution of shells with different energies $K$.

same $R_{0}$, about $30 \%$ higher than those derived neglecting the effet of the cosmological constant.

When the value of $A$ is known (we noted that the value of $A$ varies according to the considered age of the universe), Eq. (3) can be integrated for different values of $K$ or, equivalently, for different values of the initial velocity. The inclusion of the cosmological constant modifies the general picture of the LT model. The central core, in which shell crossings have already occurred, and the zero-velocity surface are still present. However, for bound shells $(K<0)$, which will reach the zerovelocity surface in the future, the turnaround occurs only if $K<K_{\mathrm{c}}=-4.06347$, where $K_{\mathrm{c}}$ corresponds to the energy for which the maximum expansion radius coincides with the zerogravity surface (ZGS). For energy values higher than the critical value $K_{\mathrm{c}}$, when the shell crosses the ZGS (located at $y_{\mathrm{c}}=1.391$ ), the acceleration is positive and there is no fallback. This behavior is illustrated in Fig. 1, where the evolution of shells with different energies $K$ is shown. The shell with $K=-6.3$ reached maximum expansion $\sim 8.0 \mathrm{Gyr}$ ago and has already collapsed. The shell with $K=-5.0$ attained maximum expansion $\sim 3.8 \mathrm{Gyr}$ ago and is still collapsing. Galaxies identified presently with such a shell have negative velocities. The shell with the particular energy $K=-4.4683$ has just reached maximum expansion or the zero-velocity surface and will collapse completely within $\sim 13.8$ Gyr from now. Finally, for the shell with $K=-4.06$, the zero-velocity radius is just beyond the ZGS and the collapse will never occur.

A fit of our numerical solution provides the present velocity-distance relation

$v(R)=-\frac{0.976 H_{0}}{R^{n}}\left(\frac{G M}{H_{0}^{2}}\right)^{(n+1) / 3}+1.377 H_{0} R$

with $n=0.627$. Using the definition and value of $A$, it is trivial to show that the equation above satisfies the condition $v\left(R_{0}\right)=0$.

This equation was tested by PP06 on a group of galaxies extracted from cosmological simulations whose properties were similar to those of Local Group. A fit of Eq. (7) to simulated data allows us to recover quite confidently the mass of the central pair of galaxies.

\subsection{Angular momentum effects}

If orbits are not purely radial, the equation of motion (Eq. (1)) becomes

$\frac{\mathrm{d}^{2} R}{\mathrm{~d} t^{2}}=-\frac{G M}{R^{2}}+\Omega_{\Lambda} H_{0}^{2} R+\frac{j^{2}}{R^{3}}$,
Table 1. Angular momentum effects on constants and fitting parameters.

\begin{tabular}{lllll}
\hline \hline$K_{J}$ & $b$ & $n$ & $A$ & $y_{\mathrm{ZG}}$ \\
\hline 0.0 & 1.377 & 0.627 & 3.7683 & 1.391 \\
0.1 & 1.319 & 0.690 & 3.9516 & 1.379 \\
1.0 & 1.156 & 0.900 & 5.6353 & 1.296 \\
\hline
\end{tabular}

where $j$ is the specific angular momentum of the shell. Numerical simulations indicate that the specific angular momentum is described well by a power law, e.g. $j=\kappa R^{\alpha}$ with $\alpha=1.1 \pm 0.3$ (Bullock et al. 2001). The coefficient $\kappa$ varies with time, reflecting the halo mass accretion history. To study the effects of angular momentum, we assume that $\alpha=1$ and $\kappa$ to be a constant. Under these conditions, in dimensionless variables, the equation of motion can be written as

$\frac{\mathrm{d}^{2} y}{\mathrm{~d} x^{2}}=-\frac{A}{2 y^{2}}+\Omega_{\Lambda} y+\frac{K_{J}}{y}$

where $K_{J}=\left(\kappa / H_{0} R_{0}\right)^{2}$. The equation above was integrated by using the same procedure as before, for $K_{J}=0.1$ and $K_{J}=1.0$. If the dimensionless velocity profile is fitted, as previously, by the relation $u=-b / y^{n}+b y$, the resulting parameters for the different values of $K_{J}$ are given in Table 1. For comparison, the corresponding values for the constant $A$ and the zero-gravity surface $y_{\mathrm{ZG}}$ are also given.

A simple analysis of Table 1 indicates that, as expected, the inclusion of the "centrifugal" force term steepens the velocity profile and decreases the radius of the zero-gravity surface. Moreover, the required value of the constant $A$ increases for higher values of the angular momentum and, as a consequence, for a given $R_{0}$ the derived masses are still more important than those derived from the modified or the "canonical" TL model.

\section{Application to nearby groups}

A significant amount of data on nearby groups have been obtained by different observers, in particular by Karachentsev and collaborators. New dwarf galaxies have been discovered as a consequence of searches on the POSS II and ESO/SERC plates (Karachentseva \& Karachentsev 1998, 2000) as well as on "blind" HI surveys (Kilborn et al. 2002). Distances to individual members of nearby groups have been derived from magnitudes of the tip of the red giant branch (Karachentsev 2005 and references therein), which have enabled more robust membership assignments and more accurate dynamical analyses to be made.

To apply Eq. (7), distances and velocities of each galaxy should be computed with respect to the center of mass. The radial distance $R$ is simply given by

$R^{2}=D^{2}+D_{\mathrm{g}}^{2}-2 D D_{\mathrm{g}} \cos \theta$

where $D$ is the distance to the center of mass, $D_{\mathrm{g}}$ is the distance to the considered galaxy, and $\theta$ is the angular separation between the galaxy and the center of mass. Observed velocities are generally given in the heliocentric system and were converted into the Local Group rest frame using the prescriptions of the RC2 calalog. If $V$ and $V_{\mathrm{g}}$ are the velocities of the center of mass and of the galaxy with respect to the LG rest frame respectively, the velocity difference along the radial direction between both objects is

$V(R)=V_{\mathrm{g}} \cos \alpha-V \cos \beta$, 
where $\beta=\theta+\alpha$ and $\tan \alpha=D \sin \theta /\left(D_{\mathrm{g}}-D \cos \theta\right)$. The final list of galaxies within each group excludes objects of uncertain distances and/or velocities as well as objects beyond the zero energy surface, which are supposed to be unbound. The latter are chosen after a first analysis in which the zero energy radius is estimated roughly for each group. Finally, for all considered galaxies, we affected an error equal to $10 \%$ of the respective values to both their radial distances $R$ and velocities $V$. It is worth mentioning that increasing this latter value up to $20 \%$ does not affect significantly the estimations of $H_{0}$ and the mass $M$ of each studied group.

\subsection{The M81 group}

Karachentsev et al. (2002a) presented a detailed study of the M81 complex. A distance of $3.5 \mathrm{Mpc}$ was estimated from the brightness of the tip of the red giant branch, based on HST/WFPC2 images of different members of the association. Using distances and radial velocities of about 50 galaxies in and around the M81 complex, Karachentsev et al. (2002a) estimated the radius of the zero-velocity surface to be $R_{0}=(1.05 \pm 0.07) \mathrm{Mpc}$ and, using the LT model, derived a total mass within $R_{0}$ equal to $(1.6 \pm 0.3) \times 10^{12} M_{\odot}$. Karachentsev \& Kashibadze (2006) found a slightly lower value for the radius of the zero-velocity surface around the pair M81/M82, i.e. $R_{0}=(0.89 \pm 0.05) \mathrm{Mpc}$, corresponding to a total mass inside $R_{0}$ of $(1.03 \pm 0.17) \times 10^{12} M_{\odot}$.

As explained in PP06, our analysis follows a different approach. We performed a non-linear fit of Eq. (7) to the available data, searching for the values of both the mass inside $R_{0}$ and the Hubble constant, which minimized the scatter. This procedure for the M81 complex provides a total mass of $(9.2 \pm 2.4) \times 10^{11} M_{\odot}$, which is consistent with the revised value by Karachentsev \& Kashibadze (2005). The derived value of the Hubble constant (in units of $100 \mathrm{~km} \mathrm{~s}^{-1} \mathrm{Mpc}^{-1}$ ) is $h=$ $0.67 \pm 0.04$. We emphasize that the quoted errors are estimates based on the spread of values derived from the fitting procedure and are not formal statistical errors. Figure $2 \mathrm{a}$ shows the velocity-distance relation based on these data. Solid points are actual data and the solid curve is the best fit solution of Eq. (7).

\subsection{The CenA/M83 complex}

Direct imaging of dwarf galaxies in the Centaurus A (NGC 5128) group were obtained by Karachentsev et al. (2002b, 2007). They showed that these galaxies are concentrated around Cen A and M83 (NGC 5236) and that their distances to the Local Group were $3.8 \mathrm{Mpc}$ and $4.8 \mathrm{Mpc}$ respectively. Using velocities and distances of individual members, the radius of the zero-velocity surface around Cen $\mathrm{A}$ was estimated to be $R_{0}=(1.44 \pm 0.13) \mathrm{Mpc}$, corresponding to a total mass inside $R_{0}$ of $(6.4 \pm 1.8) \times 10^{12} M_{\odot}$. According to the authors, effects of the cosmological constant were taken into account. Woodley (2006) also performed a dynamical investigation of the Cen A group and, based on different mass indicators, he estimated a mass for the Cen A groups of $(9.2 \pm 3.0) \times 10^{12} M_{\odot}$.

Figure $2 \mathrm{~b}$ shows the velocity-distance diagram based on the available data. The solid line represents again the best fit solution of Eq. (7). For Cen A, our analysis provides a mass of $(2.1 \pm 0.5) \times 10^{12} M_{\odot}$, which is a factor 3-4 lower than the aforementioned estimates. We discuss later the consequences of these mass determinations. The value of the Hubble parameter estimated by the fitting procedure is $h=0.57 \pm 0.04$.
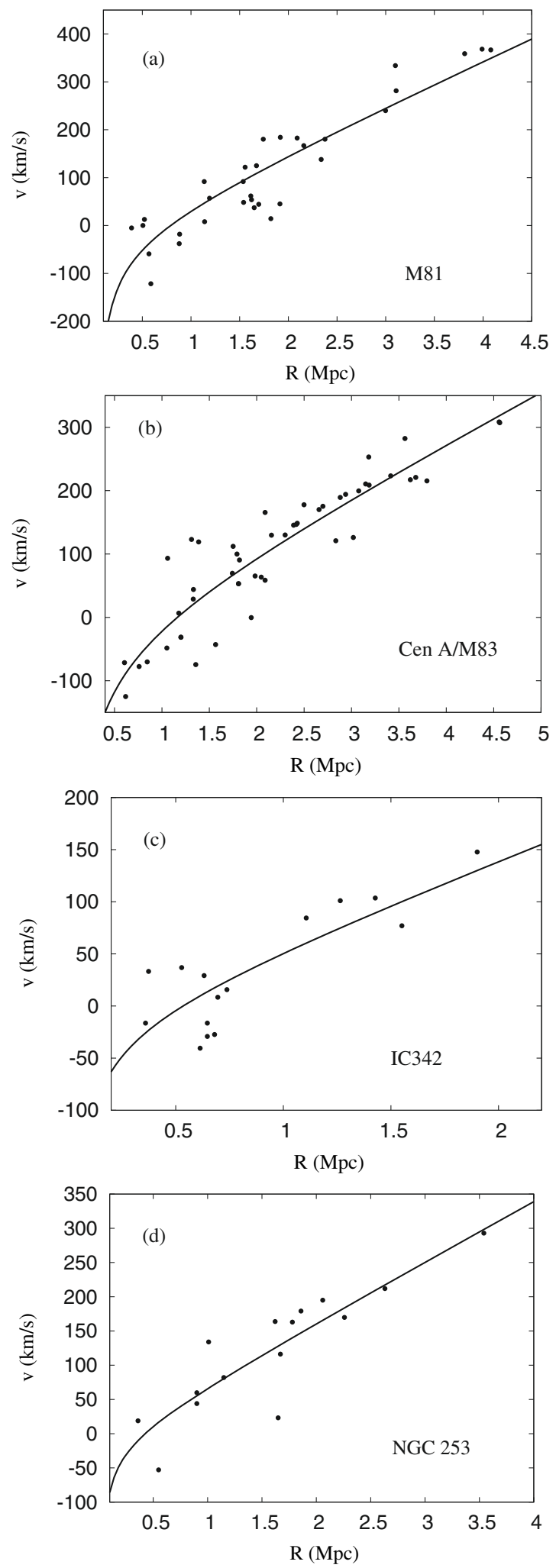

Fig. 2. Velocity-distance diagrams based on available data relative to a) the M81 group, b) the CenA/M83 complex, c) the IC342/Maffei-I group and d) the NGC 253 group. Solid lines are best fit to Eq. (7).

\subsection{The IC342/Maffei-I group}

An investigation of these groups was performed by Karachentsev et al. (2003a). They found that seven dwarf galaxies are associated with the IC342 group, at an average distance of $3.3 \mathrm{Mpc}$ from the Local Group. The Maffei-I 
association consists of about eight galaxies with an uncertain distance estimate of about $3 \mathrm{Mpc}$. According to Karachentsev et al. (2003a), the total mass of this complex inside the zerovelocity surface $R_{0}=(0.9 \pm 0.1) \mathrm{Mpc}$ is $(1.07 \pm 0.33) \times 10^{12} M_{\odot}$, a value that agrees with virial estimates, according to those authors.

Our own analysis of the same data leads to a total mass that is about a factor of 5 smaller, namely, $(2.0 \pm 1.3) \times 10^{11} M_{\odot}$. The best fit of the velocity-distance relationship (solid curve) to data (solid points) is shown in Fig. 2c. Our results suggest that the zero-velocity surface has a radius $R_{0} \simeq 0.53 \mathrm{Mpc}$ and can hardly be as high as the value given by Karachentsev et al. (2003a), as a simple inspection of our plot indicates. The simultaneous estimate of the Hubble constant from these data is $h=0.57 \pm 0.10$.

\subsection{The NGC 253 (Sculptor) group}

This association was studied by Karachentsev et al. (2003b), who described the system as small, loose concentrations of galaxies around NGC 300, NGC 253, and NGC 7793. The authors estimated the zero-velocity radius to be $R_{0}=0.7 \pm 0.1 \mathrm{Mpc}$ and a total mass of $(5.5 \pm 2.2) \times 10^{11} M_{\odot}$.

The non-linear fit of Eq. (7) to the data (Fig. 2d) provides a total mass of $(1.3 \pm 1.8) \times 10^{11} M_{\odot}$ and the large uncertainty suggests that the data is probably incomplete, in particular for objects with negative velocities (falling into the core). The derived Hubble constant is $h=0.63 \pm 0.06$, whose determination is less affected by the absence of galaxies with negative velocities.

We have revisited our previous analysis of the Virgo cluster. To be consistent with the hypothesis of our model, only galaxies with Virgocentric distances higher than 3.5 Mpc were selected, since most of the cluster mass is contained inside a sphere of $\sim 7^{\circ}$ radius $(\sim 2.2 \mathrm{Mpc})$. The total luminosity of the cluster, $L=2.4 \times 10^{12} L_{B, \odot}$, was taken from Sandage et al. (1985).

\subsection{Masses and $M / L$ ratios}

Table 2 summarizes our mass estimates, including revised values for the Local Group and the Virgo cluster derived in our previous work (PP06). Mass-to-light ratios were computed by using photometric data from both $\mathrm{NED}^{1}$ and $\mathrm{LEDA}^{2}$ databases. Asymptotic magnitudes for a given object in both databases sometimes disagree. For instance, in the case of IC 342, NED indicates $B=11.24$, whereas LEDA provides $B=9.10$. This is an extreme case, but differences in the range of $0.3-0.5 \mathrm{mag}$ are present for some objects. Because of these uncertainties, we adopted the average $B$-luminosity derived from asymptotic magnitudes and reddening given in both databases, and the distances mentioned above. We note that luminosities derived by such a procedure are, on average, smaller by a factor of two than those given for instance by Karachentsev (2005), which explains the differences between his $M / L$ ratio values and our present estimates. Therefore, uncertainties in the $M / L$ ratios reflect uncertainties in the mass determination, asymptotic magnitudes, and distances. Simple inspection of these numbers indicates that the $M / L$ ratio increases with the more massive systems, a well known behavior.

\footnotetext{
1 http://nedwww.ipac.caltech.edu

2 http://leda.univ-lyon $1 . f r$
}

Table 2. Properties of Groups: masses are in units of $10^{12} M_{\odot}$ and massto-light ratios are in solar units for the $B$-band. Columns $2-3$ correspond to the modified LT model, while the rightmost column provides masses derived for the "canonical" LT model.

\begin{tabular}{llll}
\hline \hline Group & Mass $\left(\Omega_{\Lambda}=0.7\right)$ & $M / L$ & Mass $\left(\Omega_{\Lambda}=0\right)$ \\
\hline M31/MW & $2.4 \pm 0.8$ & $60 \pm 20$ & $2.5 \pm 0.8$ \\
M81 & $0.92 \pm 0.24$ & $56 \pm 20$ & $1.3 \pm 0.4$ \\
NGC 253 & $0.13 \pm 0.18$ & $9 \pm 8$ & $0.12 \pm 0.18$ \\
IC 342 & $0.20 \pm 0.13$ & $10 \pm 8$ & $0.22 \pm 0.16$ \\
CenA/M83 & $2.1 \pm 0.5$ & $51 \pm 20$ & $2.2 \pm 0.6$ \\
Virgo & $1400 \pm 300$ & $580 \pm 106$ & $1800 \pm 400$ \\
\hline
\end{tabular}

\subsection{Effects of the cosmological constant}

How does the cosmological constant affects the results? The original $M-R_{0}$ relation derived from the LT model is

$M=\frac{\pi^{2}}{8 G} \frac{R_{0}^{3}}{T_{0}^{2}}$

where $T_{0}$ is the age of the universe. For a flat $\Lambda C D M$ cosmology, $T_{0}=g(\Omega) / H_{0}$ where

$g(\Omega)=\int_{0}^{\infty} \frac{d z}{(1+z) \sqrt{\Omega_{\Lambda}+\Omega_{\mathrm{m}}(1+z)^{3}}}$.

Adopting $\Omega_{\mathrm{m}}=0.3$, one obtains $g(\Omega)=0.964$ and, by substituting the corresponding age in Eq. (12), one obtains a numerical coefficient about 30\% smaller than Eq. (6), as PP06 already emphasized. In the "canonical" LT model, the cosmological constant therefore affects only the age determination and, for a given value of $R_{0}$, the corresponding masses are smaller by that factor in comparison with masses derived from Eq. (6).

Another approach, adopted by PP06 and in the present work, consists of fitting the theoretical velocity-distance relation to data and deriving consistently the total mass and Hubble parameter. In this case, to analyze the effects of the cosmological constant we have to compare the predictions for both models. The $v-R$ relation for the "canonical" TL model is

$v(R)=-1.038\left(\frac{G M}{R}\right)^{1 / 2}+1.196 H_{0} R$.

We note that for $v\left(R_{0}\right)=0$, Eq. (12) with the correct numerical coefficient is recovered. In Fig. 3 , the $v-R$ relation in dimensionless variables is shown either for the "canonical" LT model (Eq. (14)) or the modified LT model (Eq. (7)). For radial distances smaller than $R_{0}$, the modified LT model provides higher negative velocities than the "canonical" LT model, a consequence of an earlier turnaround. For distances longer than $y \sim 1.39$, the acceleration becomes positive and velocities are slightly higher for the modified LT model, reducing both the distance within which the outer shells are gravitationally bound and, consequently the distance at which the expanding shells merge with the Hubble flow.

The first point to emphasize is that the fit qualities (measured by the $\chi^{2}$ values) of both $v-R$ relations (Eqs. (7), (14)) to data is comparable. The corresponding dispersion velocities of the peculiar motion over the Hubble flow are therefore practically the same for both models and are given in the last column of Table 3. Excluding the Virgo cluster, the mean value of the dispersion velocity for the other five groups is $\sigma_{1 \mathrm{D}}=43 \pm 7 \mathrm{~km} \mathrm{~s}^{-1}$, in agreement with the previous estimates. 


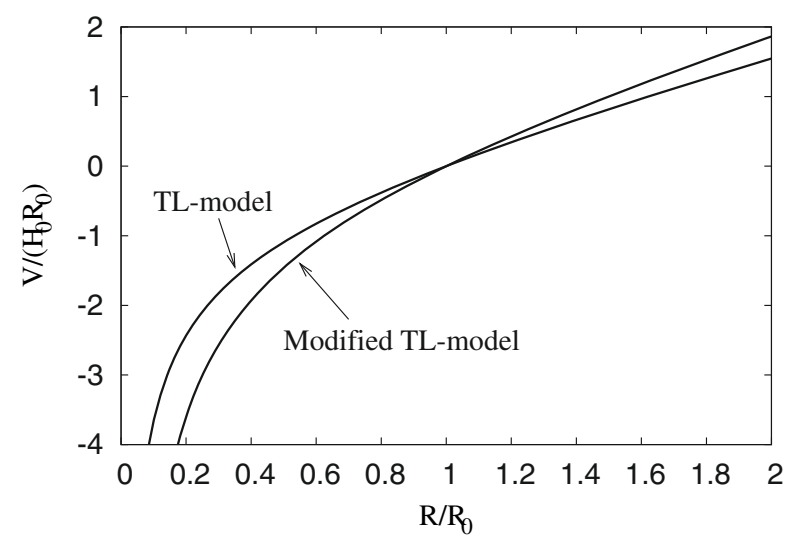

Fig. 3. Comparison between the $v-R$ relation (dimensionless variables) derived from the "canonical" LT model (Eq. (14)) and from the modified LT model (Eq. (7)).

Table 3. The Hubble parameter derived from local flows: the second column corresponds to values derived from the modified TL model, while the third column corresponds to the "canonical" TL model. The last column provides the velocity dispersion from the fit of data to the $v-R$ relation for the modified TL model. The mean value of the velocity dispersion excludes the Virgo cluster.

\begin{tabular}{llll}
\hline \hline Group & $h\left(\Omega_{\Lambda}=0.7\right)$ & $h($ TL model $)$ & $\sigma\left(\mathrm{km} \mathrm{s}^{-1}\right)$ \\
\hline M31/MW & $0.73 \pm 0.04$ & $0.87 \pm 0.05$ & 38 \\
M81 & $0.67 \pm 0.04$ & $0.82 \pm 0.05$ & 53 \\
NGC 253 & $0.63 \pm 0.06$ & $0.74 \pm 0.08$ & 45 \\
IC 342 & $0.57 \pm 0.10$ & $0.68 \pm 0.12$ & 34 \\
CenA/M83 & $0.57 \pm 0.04$ & $0.68 \pm 0.04$ & 45 \\
Virgo & $0.71 \pm 0.09$ & $0.92 \pm 0.12$ & 345 \\
\hline mean & $0.65 \pm 0.07$ & $0.79 \pm 0.10$ & $43 \pm 7$ \\
\hline
\end{tabular}

Masses derived from the "canonical" LT model are given in the last column of Table 2 and they differ, on average, by $\sim 10 \%$ from the values estimated from the modified LT model. It is worth mentioning that such a difference is less than that expected by the use either of Eq. (6) or Eq. (12). In this procedure, the radius of the zero-velocity surface is determined independently and, as PP06 have shown, in this case, the masses calculated by the modified LT model are about 30\% higher than those derived by the "canonical" model. By adopting the nonlinear fit of the $v-R$ relation, the parameters were optimized and the value of $R_{0}$ was not the same for both models but the masses were comparable. Such a method also permits an "optimized" estimate of the Hubble parameter, given in columns two (modified LT model) and three ("canonical" LT model) of Table 3 respectively. Inspection of these figures reveals that the Hubble parameter estimated from the fit of Eq. (14) is systematically higher, by about 33\%, than those derived from the fit of Eq. (7).

Analysis of the 3-year data of WMAP (Spergel et al. 2007) measured the Hubble parameter $h=0.73 \pm 0.03$. However, studies of the local expansion flow calculate smaller values. From the analysis of 25 galaxies with velocities less than $500 \mathrm{~km} \mathrm{~s}^{-1}$, Karachentsev et al. (2006) derived $h=0.68 \pm 0.15$ and Sandage et al. (2006), from a recalibration of distance indicators, obtained $h=0.62 \pm 0.05$. The mean values shown in Table 3 for both models are consistent with these determinations within the estimated uncertainties, although the mean value of $H_{0}$ derived from the "canonical" TL model corresponds to an age for the universe of $T_{0} \simeq 12.2 \mathrm{Gyr}$, which appears to be very low.

\section{Conclusions}

Modified by inclusion of a cosmological constant, the velocity profile of the LT model has been calculated. The inclusion of this term in the equation of motion modifies some characteristics of the "canonical" LT model. Shells inside the zero-velocity surface collapse earlier and, as a consequence, at a given distance, negative velocities of larger amplitude than those derived from the "canonical" model are obtained. Shells, whose maximum expansion radius is beyond the critical value $R \sim 1.39 R_{0}$, will never collapse, since their acceleration becomes positive.

Data for dwarf galaxies belonging to nearby groups and galaxies in the outskirts of the Virgo cluster are described well by such a model, indicating that these objects are either collapsing or expanding, to collapse again in the future, if their distances are smaller than $\sim 1.39 R_{0}$. Such good agreement between the theoretical $v-R$ relation and the data implies that the use of the virial theorem to estimate the core masses is questionable.

However, the $v-R$ relation derived from the "canonical" LT model provides an equally acceptable fit and the present results cannot be used as an argument in favor of the detection of effects due to the cosmological constant on scales of the order of few Mpc. Core masses derived from both models agree to within $10 \%$ but the Hubble parameter estimated from the "canonical" LT model is systematically higher than values produced by the modified model. Nevertheless, mean values are compatible with other independent estimates.

The mean value of the 1D dispersion velocity derived from the five groups (Virgo excluded) investigated in this work is $43 \pm 7 \mathrm{~km} \mathrm{~s}^{-1}$, smaller than values derived from cosmological simulations within scales of $1-3 \mathrm{Mpc}$, i.e. $73 \mathrm{~km} \mathrm{~s}^{-1}$ (PP06) and $80 \mathrm{~km} \mathrm{~s}^{-1}$ (Macciò et al. 2005). It is worth mentioning that Axenides \& Perivolaropoulos (2002) studied the dark energy effects in the growth of matter fluctuations in a flat universe. They concluded that the dark energy can indeed cool the local Hubble flow but the required parameters to make the predicted dispersion velocity of the order of $40 \mathrm{~km} \mathrm{~s}^{-1}$ are ruled out by observations that constrain either the present dark energy density or the equation of state parameter $w\left(=P_{x} / \varepsilon_{x}\right)$. Thus, the dark energy with a time independent equation of state cannot explain the observed quietness of the local Hubble flow, which remains an enigma.

Acknowledgements. S.P. acknowledges the financial support through a ANR grant. We thank the referee for his useful comments that helped to improve the text of this paper.

\section{References}

Axenides, M., \& Perivolaropoulos, L. 2002, Phys. Rev. D, 65, 127301

Bullock, J. S., Dekel, A., Kolatt, T. S., et al. 2001, ApJ, 555, 240

Chernin, A. 2001, Phys. Usp., 44, 1099

Ekholm, T., Baryshev, Y., Teerikorpi, P., Hanski, M. O., \& Paturel, G. 2001, A\&A, 368, L17

Geller, M. J., \& Huchra, J. P. 1983, ApJS, 52,61

Giraud, E. 1986, A\&A, 170, 1

Governato, F., Moore, B., Cen, R., Stadel, J., Lake, G., \& Quinn, T. 1997, New Astron., 2, 91

Gunn, J. E., \& Gott, J. R. I., 1972, ApJ, 176, 1

Hoffman, G. L., Olson, D. W., \& Salpeter, E. E. 1980, ApJ, 242, 861

Hoffman, Y., Martinez-Vaquero, L. A., Yepes, G., \& Gottlöber, S. 2008, MNRAS, 386, 390

Huchra, J. P., \& Geller, M. J. 1982, ApJ, 257, 423

Karachentsev, I. D. 2005, AJ, 129, 178 
Karachentsev, I. D., \& Kashibadze, O. G. 2006, Astrophysics, 49, 3

Karachentsev, I. D., Dolphin, A. E., Geisler, D., et al. 2002a, A\&A, 383, 125

Karachentsev, I. D., Sharina, M. E., Dolphin, A. E., et al. 2002b, A\&A, 385, 21

Karachentsev, I. D., Sharina, M. E., Dolphin, A. E., \& Grebel, E. K. 2003a, A\&A, 408, 111

Karachentsev, I. D., Grebel, E. D., Sharina, M. E., et al. 2003b, A\&A, 404, 93

Karachentsev, I. D., Dolphin, A., Tully, R. B., et al. 2006, AJ, 131, 1361

Karachentsev, I. D., Tully, R. B., Dolphin, A., et al. 2007, AJ, 133, 504

Karachentseva, V. E., \& Karachentsev, I. D. 1998, A\&A, 127, 409

Karachentseva, V. E., \& Karachentsev, I. D. 2000, A\&AS, 146, 359

Kilborn, V. A., Webster, R. L., Staveley-Smith, L., et al. 2002, AJ, 124, 690

Lahav, O., Lilje, P. B., Primack, J. R., \& Rees, M. J. 1991, MNRAS, 251, 128

Lemaître, G. 1933, Ann. Soc. Sci. Bruxelles, A53, 51

Lynden-Bell, D. 1981, The Observatory, 101, 111

Macciò, A. V., Governato, F., \& Horellou, C. 2005, MNRAS, 359, 941

Maor, I., \& Lahav, O. 2005, JCAP Physics, 7, 3

Niemi, S.-M., Nurmi, P., Heinämäki, P., \& Valtonen, M. 2007, MNRAS, 382, 1864
Nolthenius, R., \& White, S. D. M. 1987, MNRAS, 225, 505

Olson, D. W., \& Silk, J. 1979, ApJ, 233, 395

Peebles, P. J. E. 1990, ApJ, 362, 1

Peirani, S., \& de Freitas Pacheco, J. A. 2006, New Astron., 11, 325 (PP06)

Sandage, A. 1986, ApJ, 307, 1

Sandage, A. \& Tammann, G. A. 1975, ApJ, 196, 313

Sandage, A., Bingelli, B., \& Tammann, A. 1985, AJ, 90, 1759

Sandage, A., Tammann, G. A., Saha, A., et al. 2006, ApJ, 653, 843

Schlegel, D., Davis, M., Summers, F., \& Holtzman, J. A. 1994, ApJ, 427, 527

Spergel, D. N., Bean, R., Doré, O., et al. 2007, ApJS, 170, 377

Teerikorpi, P., Bottinelli, L., Gouguenheim, L., \& Paturel, G. 1992, A\&A, 260, 17

Teerikorpi, P., Chernin, A. D., \& Baryshev, Y. V. 2005, A\&A, 440, 791

Tolman, R. C. 1934, Proc. Nat. Acad. Sci., 20, 169

Tully, R. B., \& Shaya, E. J. 1984, ApJ, 281, 31

Wang, L., \& Steinhardt, P. J. 1998, ApJ, 508, 483

Woodley, K. A. 2006, AJ, 132, 2424 